\title{
Electromyographic analysis of transversus abdominis/internal oblique musscles during the execution of pelvic patterns of proprioceptive neuromuscular facilitation
}

\author{
Josepha Karinne de Oliveira Ferro ${ }^{1}$ \\ José Vicente Pereira Martins ${ }^{2}$ \\ Bruna Rhayane da Cunha Melo Ribeiro ${ }^{1}$ \\ Thaís de Siqueira Manta ${ }^{1}$ \\ Andrea Lemos ${ }^{1}$ \\ Keytte Camilla Souza de Amorim ${ }^{1}$ \\ Paulo José Moté Barboza ${ }^{3}$ \\ Alberto Galvão de Moura Filho ${ }^{1}$ \\ Daniella Araújo de Oliveira ${ }^{1}$
}

\footnotetext{
${ }^{1}$ Department of Physical Therapy, Health Sciences Center, Federal University of Pernambuco, Recife, Pernambuco, Brazil.

2 Department of Physical Therapy, Health Sciences Center, Federal University of Rio de Janeiro, Rio de Janeiro, Rio de Janeiro, Brazil.

${ }^{3}$ Physical Therapist at Integrated Rehabilitation and Aquatic Therapy Center (CIRTA), Rio de Janeiro, Rio de Janeiro, Brazil.
}

\section{$\triangle$}

Josepha Karinne de Oliveira Ferro Rua Amália Bernardino de Souza 264, 1601 - Boa Viagem, Recife Pernambuco, 51021-150

Phone: +5581998058006 karinneferro@gmail.com

This article was edited by: Marcelo Moraes Valença Juliana Ramos de Andrade

\section{Keywords:}

Electromyography

Abdominal Muscles

Muscle Stretching Exercises

Proprioceptive Neuromuscular

Facilitation (PNF) Stretching

\begin{abstract}
Objective To evaluate the electromyographic response of the transversus abdominis/internal oblique muscles $(\mathrm{TrA} / \mathrm{IO})$ during the execution of the four pelvic patterns of proprioceptive neuromuscular facilitation (PNF).

Methods Cross-sectional study. Were evaluated 21 women aged $18-38$ years. The right TrA/IO complex, ipsilateral to the execution of a PNF combination of isotonics technique was monitored by surface electromyography. Three repetitions were performed with two-minute intervals between them in the four PNF pelvic patterns: anterior elevation, posterior depression, anterior depression, and posterior elevation. For the analysis of the electromyographic signal, a period of $500 \mathrm{~ms}$ adjusted to the central value was extracted and the Root Mean Square amplitude was analyzed. Descriptive statistics and ANOVA test was used with a $95 \%$ confidence interval.

Results There was a higher TrA/IO activity in the concentric phase in the anterior elevation pattern ( 36.2 $\pm 32.3 \mu \mathrm{V})$ when compared to previous depression $(19.5 \pm 12.9 \mu \mathrm{V})$, posterior elevation $(16.1 \pm 8.7 \mu \mathrm{V})$, posterior depression $14.6 \pm 5.9 \mu \mathrm{V})$. In addition, in the antero-elevation there is greater activation of the $\mathrm{TrA} / 1 \mathrm{O}$ muscle complex when compared to the other patterns $(p<0.01)$.

Conclusion The higher EMG response of the $\mathrm{TrA} / \mathrm{IO}$ found in the anterior elevation pattern reveals its usefulness for clinical use.
\end{abstract}




\section{Introduction}

The abdominal muscles play an important role in lumbar and pelvic dynamic stability, the transversus abdominis being the main stabilizing muscle being activated before the other abdominal wall muscles to protect the spine from internal and external overloads, in addition to preparing the body to perform movements and maintain postural balance. ${ }^{1,2}$

TIn order to perform these functions, an adequate neuromuscular control is necessary and the central nervous system receives afferent information from peripheral mechanoreceptors, interprets, plans and coordinates the execution of mechanisms to maintain stability and movements in response to demand. ${ }^{3}$

Proprioceptive Neuromuscular Facilitation (PNF) is a type of functional approach based on motor control and its effects on learning. Principles and procedures are used to facilitate neuromuscular response and specific techniques, depending on the goal to be achieved. Tactile stimulation and external opposition to the movement (resistance) are extremely important to improve muscular strength and hypertrophy, contributing to adaptations such as increased recruitment and synchronization of motor units, coactivation of agonists and antagonists and changes in the descending neural pathways and can be evaluated through of electromyographic records. ${ }^{4,5}$

PNF pelvic patterns promote lumbar extension and activation of the stabilizing musculature of the trunk, reducing pain. ${ }^{6}$ Among the techniques of PNF, the combination of isotonics, which seems to be one of the most effective when it is intended to increase strength and improve motor control. Promotes the activation of agonist muscles with concentric, isometric and eccentric contraction, through resistance, without relaxation, leading to the activation of this muscle in a wide and complete manner in the same intervention. . $^{5,7}$

Few studies analyze the electrophysiological measures of the muscle complex TrA/IO during the execution of the PNF pelvic patterns. This work aims to evaluate the EMG response of the muscle complex ( $\mathrm{TrA} / \mathrm{IO})$ during the execution of the four PNF pelvic patterns in the concentric, isometric and eccentric contractions of the combination of isotonics.

\section{Methods}

This is a cross-sectional study. The data were collected from September 2018 to March 2019. The Committee of Ethics and Research with Human Beings, Resolution CNS 466/12, approved it under opinion $n^{\circ} 2546842$. The volunteers who agreed to participate gave their informed consent, being assured the complete anonymity and the right to stop participating at any time of the research.
The convenience sample consisted of young women, aged 18 to 38 years, healthy, in the menacme and nulligravidas. Women in the gestational period who had previous surgeries in the pelvic and/or abdominal region, neuromuscular diseases, chronic constipation and musculoskeletal dysfunction in the pelvic region (chronic pelvic pain and pyriform syndrome) were excluded.

The convenience sample consisted of young women, aged 18 to 38 years, healthy, in the menacme and nulligravidas. Women in the gestational period who had previous surgeries in the pelvic and/or abdominal region, neuromuscular diseases, chronic constipation and musculoskeletal dysfunction in the pelvic region (chronic pelvic pain and pyriform syndrome) were excluded.

Initially, a semi-structured form containing information such as personal and sociodemographic data, anthropometric characteristics, associated diseases, practice and frequency of physical activity was applied.

Then the electromyographic activity of the abdominal muscles was recorded during the performance of the PNF pelvic patterns through the technique combination of isotonics.

Two researchers ( $A$ and $B$ ) participated in the study. Researcher A was responsible for performing the screening, explaining the research procedures and obtaining the consent. Researcher $B$ is a physiotherapist who specializes in urogynecology with training to perform the PNF pelvic patterns by an advanced PNF instructor recognized by International Proprioceptive Neuromuscular Facilitation Association (IPNFA). Both performed the recording of the electromyographic signal, while the researcher $A$ assisted in the capture of the signal, the researcher $B$ performed the PNF technique simultaneously.

\section{Recording of the electromyographic signal}

In order to capture the signal, a Miotec ${ }^{\circledR}$ surface electromyograph (model myotool 400, Miotec Equipamentos Biomédicos Ltd. Porto Alegre, Brazil) was used, which has four channels and a 14-bit analog-to-digital converter (A / D) resolution and an internal gain of 1000 times. The sampling frequency per $2000 \mathrm{~Hz}$ channel, $110 \mathrm{~dB}$ common mode rejection (CMRR), $1010 \mathrm{Ohm} / 2 \mathrm{pF}$ input impedance and 4th order Butterworth analog filter were used. The captured signal was recorded

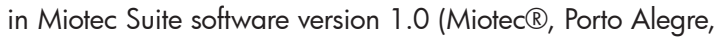
Brazil) for later analysis by Miograph ${ }^{\circledR}$ software (Miotec suite ${ }^{\circledR}$ version 1.0).

Signal capture occurred during the execution of PNF pelvic patterns. The electromyographic recording followed the recommendations of the International Society of Electrophysiology and Kinesiology. ${ }^{8}$ Using low-impedance (25 mm diameter) sel- 
f-adhesive and disposable circular surface electrodes oriented along the muscle fibers in the action line of the complex (TrA/ IO) of the right side abdomen, being positioned $2 \mathrm{~cm}$ proximal to the midpoint between the anterosuperior iliac spine and the pubic symphysis. ${ }^{9}$ Prior to registration, wipes were wiped with moist tissues from the region where the electrodes would be positioned, drying them immediately to reduce the impedance of the skin. Due to the placement of the therapist's hand on the iliac crest, the reference electrode was positioned in the manubrium of the sternum.

The mean peak value of three maximum voluntary contractions (MVC) was calculated. The volunteer was asked to perform the maximum possible contraction, sustained for three seconds, through an abdominal retraction, anterior flexion of the lateral trunk, isometric, for the muscle complex TrA/IO. Then, the four pelvic patterns were recorded through the technique of combining isotonic with two concentric phases.

For electromyographic analysis, a time of 500 milliseconds (ms) adjusted to its central value was extracted in order to reduce artifacts and to analyze exactly the mean of the contraction in each phase, reducing the interference of the phase changes. Root Mean Square (RMS) contraction values were recorded using the following filters: high pass $20 \mathrm{~Hz}$, low pass 500 $\mathrm{Hz}$, notch $60 \mathrm{~Hz}$.

\section{The protocol of proprioceptive neuromuscular facilitation}

Initially, researcher B, through rhythmic initiation, explained the movements that would be performed during the technique in the four pelvic patterns, always keeping the verbal command. Thus, first passive movements were performed, followed by active assisted movement and resistance movement simulating the patterns that were later employed. Finally, the volunteer was asked to perform the active movement.

In the capture of the electromyographic signal during the execution of the four pelvic patterns: anterior elevation, posterior depression, posterior elevation and anterior depression, randomized to each volunteer. The combination of isotonics technique is a sequence of concentrics, isometrics, and eccentric contractions with no rest between contractions. The protocol for this study was composed of a sequence of five different contractions being a concentric, an isometric, an eccentric succeeded by the isometric post eccentric and ending with another concentric. This contraction sequence was repeated three times consecutively, totaling four concentric phases and using the standardized verbal resistance and stimulus throughout the execution. Each phase lasted five seconds. Three records per the pelvic pattern of each volunteer were performed, with a one-minute rest interval between the repetitions and two minutes between each pattern.
The volunteer was positioned in left lateral decubitus, in body alignment, with knees at approximately $70^{\circ}$ of flexion, hip in semiflexion. The collection was done in a reserved environment, with only the evaluators. Researcher B was positioned diagonally to the volunteer during the procedure, which varied depending on the standard to be performed. In order to reduce the measurement bias, the resisted motion was performed by a single evaluator.

\section{Methods of statistical analysis}

The Statistical Package for Social Sciences (SPSS) version 20 software was used to analyze the data obtained and adopted a $95 \%$ confidence level. Descriptive statistics techniques were adopted, including tables and graphs and all results were presented as mean and their respective confidence intervals for the continuous data and in absolute and relative frequencies (\%) for ordinal and nominal data. The Levene test was used to analyze the homogeneity of the variances. The ANOVA test was applied with a Tukey post-test in order to compare the means of the mean contraction variable between concentric, isometric post-concentric, eccentric and isometric post-eccentric contractions during the protocol used in each standard. For this analysis, the mean of each type of contraction was used during the three consecutive repetitions.

\section{Results}

Twenty-one volunteers participated in the study. As three repetitions were performed, sixty-three electromyographic records in each PNF pelvic pattern were analyzed, totaling 252 records. The characterization of the sample can be seen in Table 1 .

Table 1. Characterization of the sample $(n=21)$ described in mean and confidence interval (95\%), and absolute and relative frequency

\begin{tabular}{|l|c|}
\hline \multicolumn{1}{|c|}{ Variables } & Mean $(95 \% \mathrm{Cl}) \mid \mathrm{n}(\%)$ \\
\hline Age (years) & $23.4(21.5-25.3)$ \\
\hline BMl (kg/m2) & $22.7(21.1-24.3)$ \\
\hline Physical Activity (yes) & $12(57.1 \%)$ \\
\hline Frequency of physical activity & $8(66.7 \%)$ \\
$2-4 /$ week & $4(33.3 \%)$ \\
\hline
\end{tabular}

$\mathrm{Cl}=95 \%$ confidence interval $; \mathrm{n}=$ sample size; $\mathrm{BMI}=$ body mass index.

The EMG response showed greater activation of the $\mathrm{TrA} / \mathrm{IO}$ during the concentric contraction. An increase in the amplitude of the electromyographic signal in the second concentric contraction was also observed, suggesting an increase in the recruitment of motor units (Figure 1). 


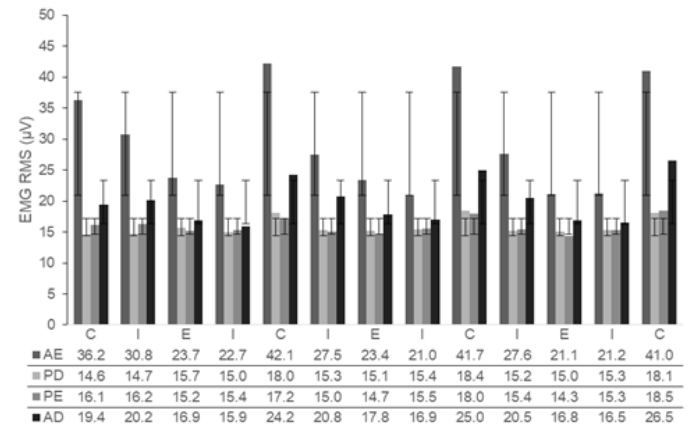

Figure 1. Mean contraction of the electromyography signal of the transversus abdominis/internal oblique muscles right side in each contraction of the combination of isotonics technique in the PNF pelvic patterns. Data described in mean \pm standard deviation ( $\mathrm{n}$ = 21) $(C=$ concentric, $1=$ isometric,$E=$ eccentric, $\mathrm{AE}=$ anterior elevation, $\mathrm{PD}=$ posterior depression, $\mathrm{AD}=$ anterior depression, $\mathrm{PE}=$ posterior elevation).

When evaluating the type of contraction in relation to the four PNF pelvic patterns, it was observed that in the anterior elevation pattern there was greater activation of the TrA/IO muscle complex when compared to the other patterns $(p<0.01)$ throughout the protocol performed (Table 2).

Table 2. Mean of contraction type of the combination of isotonics technique in the four PNF pelvic patterns $(n=21)$.

\begin{tabular}{|c|c|c|c|c|}
\hline \multicolumn{5}{|l|}{ CONCENTRIC CONTRACTION } \\
\hline & Mean \pm SD & $95 \% \mathrm{Cl}$ & $\mathrm{F}$ & p-value* \\
\hline Anterior elevation pattern (AE) & $40.2 \pm 34.8$ & $30.9-49.5$ & \multirow{4}{*}{16.6} & \multirow{4}{*}{$p<0.01$} \\
\hline $\begin{array}{l}\text { Anterior depression pattern } \\
\text { (AD) }\end{array}$ & $23.7 \pm 15.9$ & $19.5-28.0$ & & \\
\hline $\begin{array}{l}\text { Posterior depression pattern } \\
\text { (PD) }\end{array}$ & $17.2 \pm 8.6$ & $14.9-19.5$ & & \\
\hline Posterior elevation pattern (PE) & $17.4 \pm 6.8$ & $15.6-19.2$ & & \\
\hline \multicolumn{5}{|c|}{ POST-CONCENTRIC ISOMETRIC CONTRACTION } \\
\hline & Mean $\pm S D$ & $95 \% \mathrm{Cl}$ & $\mathrm{F}$ & p-value* \\
\hline Anterior elevation pattern (AE) & $28.5 \pm 20.9$ & $22.9-34.1$ & \multirow{4}{*}{13.1} & \multirow{4}{*}{$p<0.01$} \\
\hline $\begin{array}{l}\text { Anterior depression pattern } \\
\text { (AD) }\end{array}$ & $20.4 \pm 13.1$ & $16.9-23.9$ & & \\
\hline $\begin{array}{l}\text { Posterior depression pattern } \\
\text { (PD) }\end{array}$ & $15.0 \pm 5.7$ & $13.5-16.5$ & & \\
\hline Posterior elevation pattern (PE) & $15.5 \pm 5.7$ & $14.0-17.0$ & & \\
\hline \multicolumn{5}{|l|}{ ECCENTRIC CONTRACTION } \\
\hline & Mean \pm SD & $95 \% \mathrm{Cl}$ & $\mathrm{F}$ & p-value* \\
\hline Anterior elevation pattern (AE) & $22.7 \pm 15.4$ & $18.6-26.7$ & \multirow{4}{*}{8.6} & \multirow{4}{*}{$p \leq 0.01$} \\
\hline $\begin{array}{l}\text { Anterior depression pattern } \\
\text { (AD) }\end{array}$ & $17.1 \pm 7.1$ & $15.2-19.0$ & & \\
\hline $\begin{array}{l}\text { Posterior depression pattern } \\
\text { (PD) }\end{array}$ & $15.2 \pm 5.9$ & $13.7-16.8$ & & \\
\hline Posterior elevation pattern (PE) & $14.7 \pm 5.2$ & $13.3-16.1$ & & \\
\hline \multicolumn{5}{|c|}{ POST-ECCENTRIC ISOMETRIC CONTRACTION } \\
\hline & Mean \pm SD & $95 \% \mathrm{Cl}$ & $F$ & p-value* \\
\hline Anterior elevation pattern (AE) & $21.6 \pm 14.5$ & $17.7-25.4$ & \multirow{4}{*}{5.6} & \multirow{4}{*}{$p<0.01$} \\
\hline $\begin{array}{l}\text { Anterior depression pattern } \\
\text { (AD) }\end{array}$ & $16.4 \pm 7.7$ & $14.3-18.5$ & & \\
\hline $\begin{array}{l}\text { Posterior depression pattern } \\
\text { (PD) }\end{array}$ & $15.2 \pm 6.3$ & $13.5-16.8$ & & \\
\hline Posterior elevation pattern (PE) & $15.3 \pm 7.2$ & $13.4-17.2$ & & \\
\hline
\end{tabular}

*Anova test, Tukey post-test ( $A E$ in relation to $A D, P D$, and $P E)$.

\section{Discussion}

This study analyzed the EMG response of the TrA/IO muscle complex during the execution of the combination of isotonics technique in the PNF pelvic patterns, with greater muscle activation of the $\mathrm{TrA} / \mathrm{IO}$ in the anterior elevation pattern.

The transversus abdominis and the multifidus muscles play an important role in body posture in healthy individuals being activated as an anticipatory response to postural instability. ${ }^{10-12}$ During the execution of the combination of isotonics technique, manual resistance is used in the three types of contraction: concentric, isometric and eccentric in order to produce different levels of forces in each muscle contraction. ${ }^{4}$

Resistance to motion is a determinant factor for increased muscle strength, as it provides the activation of descending corticospinal pathways and increases the excitability of motoneurons, enhancing the recruitment of motor units. ${ }^{13}$ The amplitude increase of the electromyographic signal is related to increased strength and muscle recruitment in resisted exercises. ${ }^{5,14}$

Thus, we speculate that the intrinsic alterations in the control neural pathways explain the fact that, in the present study, the concentric contraction performed against manual resistance presented greater electromyographic activity.

It was also observed that in the second concentric phase there is an increase in the electromyographic signal in relation to the first concentric phase, which corresponds to the greater muscular activation in the repetition of this phase. During the repetitive training, there is an improvement in functional performance, since by improving motor control, there is greater recruitment of motor units with the consequent increase in muscle strength. $4,15,16$ We assume that this increase was due to the increase in control and motor learning, benefits of the combination of isotonics technique, organizing, at a central and medullary level, activities already present or learned by the individual.

The TrA muscle is considered the main stabilizer of the spine, besides being associated with the control of the pelvic movement. ${ }^{1}$ The pelvis is able to provide a support base for stabilizing muscles, which promote balance and assist in body transfers during activities of daily living such as walking and running due to its role in lumbar and pelvic stabilization and postural control. ${ }^{17-19}$

Because it plays an important stabilizing role in the lumbar spine, the TrA muscle has been the target of approaches during physiotherapeutic treatment in various clinical outcomes. ${ }^{19-21}$ Evidence shows the effects of proprioceptive neuromuscular facilitation on pelvic loins stability when the outcomes were 
analyzed: trunk range of motion, lumbar flexibility, abdominal muscle thickness and pain. ${ }^{6,17,22}$

A previous study using anterior elevation and posterior depression patterns when associated with scapular patterns significantly improves lumbar flexibility and pain perception in individuals with low back pain. It is believed that the response to repeated proprioceptive stimuli causes increased traction and subsequent contraction of the stabilizing muscles. ${ }^{6}$

In addition to this information, we verified, through electromyographic measurements, the activity of the $\mathrm{TrA} / \mathrm{IO}$ muscle complex in the control of the pelvis after the tactile stimulus and resistance offered during the execution of the pelvic patterns of PNF.

\section{Conclusion}

The greater activation of this muscle group occurs in the anterior elevation pattern during the combination of isotonics technique of PNF. However, new studies are needed to prove the efficacy of this standard in lumbar and pelvic stabilization, movement control, pain, balance, and functionality, and better understand its synergy with other muscles in the region.

Acknowledgments: To the Coordination for the Improvement of Higher Education Personnel (CAPES) for scholarship.

Funding Source: Josepha Karinne de Oliveira Ferro - CAPES scholarship recipient, Coordination for the Improvement of Higher Education Personnel (CAPES).

Declaration of interest: The authors report no declarations of interest.

Josepha Karinne de Oliveira Ferro

https://orcid.org/0000-0002-7643-3035

José Vicente Pereira Martins

https://orcid.org/0000-0001-9696-0265

Bruna Rhayane da Cunha Melo Ribeiro

https://orcid.org/0000-0002-4823-5859

Thaís de Siqueira Manta

https://orcid.org/0000-0001-6200-1576

Andrea Lemos

https://orcid.org/0000-0003-0631-0512

Keytte Camilla Souza de Amorim

https://orcid.org/0000-0001-7299-5308

Paulo José Moté Barboza

https://orcid.org/0000-0002-01 12-8933

Alberto Galvão de Moura Filho

https://orcid.org/0000-0002-3813-9951

Daniella Araújo de Oliveira

https://orcid.org/0000-0002-6013-978X

\section{References}

1. Paris-Alemany A, Torres-Palomino A, Marino L, CalvoLobo C, Gadea-Mateos L, La Touche R. Comparison of lumbopelvic and dynamic stability between dancers and non-dancers. Phys Ther Sport. 2018; 33: 33-39. doi:10.1016/i.ptsp.2018.06.010

2. Morales CR, Sanz DR, Reguera M de la C, Martínez SF, González PT, Pascual BM. Proprioceptive Stabili-
zerTM training of the abdominal wall muscles in healthy subjects: a quasi-experimental study. Rev Assoc Med Bras. 2018; 64 (12): 1134-1138. doi: 10.1590/18069282.64.12.1134

3. Hodges PW, Moseley GL. Pain and motor control of the lumbopelvic region: Effect and possible mechanisms. J Electromyogr Kinesiol. 2003; 13(4):361-70. doi:10.1016/S1050-6411(03)00042-7

4. Smedes F, Heidmann M, Schäfer C, Fischer N, Stępień A. The proprioceptive neuromuscular facilitation-concept; the state of the evidence, a narrative review. Phys Ther Rev. 2016; 21 (1): 17-31. doi:10.1080/10833 196.2016.1216764

5. Pin A. Facilitação Neuromuscular Proprioceptiva nas alterações musculares : conversão de fibras muscu lares vista através de eletromiografia de superfície e dinamometria. Novas Edições Acadêmicas; 2016. Disponível em: https://www.researchgate.net/ publication/291332079_Facilitacao_Neuromuscular_Proprioceptiva_nas_alteracoes_musculares_conversao_de_fibras_musculares_vista_atraves_de_eletromiografia_de_superficie_e_dinamometria [Acesso em 02 de julho de 2019].

6. Park K, Seo K. The Effects on the Pain Index and Lumbar Flexibility of Obese Patients with Low Back Pain after PNF Scapular and PNF Pelvic Patterns. J Phys Ther Sci. 2014; 26 (10): 1571-1574. doi:10.1589/ ipts.26.1571

7. Douglas J, Pearson S, Ross A, McGuigan M. Chronic Adaptations to Eccentric Training: A Systematic Review. Sport Med. 2017; 47 (5): 917-941. doi:10.1007/ s40279-016-0628-4

8. Merletti R. Standa ards for Reporting EMG Data. J Electromyogr Kinesiol. 1999; 9 (1): 3-4. doi:10.1016/ S1050-6411(97)90001-8

9. Neumann P, Gill V. Pelvic floor and abdominal muscle interaction: EMG activity and intra-abdominal pressure. Int Urogynecol J Pelvic Floor Dysfunct. 2002; 13 (2): 125-32. doi: 10.1007/s001920200027

10. Tsao H, Hodges PW. Immediate changes in feedforward postural adjustments following voluntary motor training. Exp Brain Res. 2007; 181: 537-546. doi:10.1007/ s00221-007-0950-z

11. Moseley GL, Hodges PW, Gandevia SC. Deep and Superficial Fibers of the Lumbar Multifidus Muscle Are Differentially Active During Voluntary Arm Movements. Spine (Phila Pa 1976). 2002; 27 (2): E29-36. doi: 10.1097/00007632-200201150-00013

12. Hodges PW, Richardson CA. Feedforward contraction of transversus abdominis is not influenced by the direction of arm movement. Exp Brain Res. 1997; 114 (2): 362 70. doi:10.1007/PL00005644

13. Gabriel DA, Kamen G, Frost G. Neural adaptations to resistive exercise: Mechanisms and recommendations for training practices. Sport Med. 2006; 36 (2): 133-49. doi:10.2165/00007256-200636020-00004

14. Arjunan SP, Kumar DK, Naik G. Computation and Evaluation of Features of Surface Electromyogram to Identify the Force of Muscle Contraction and Muscle Fatigue. Biomed Res Int. 2014. doi:10.1155/2014/197960

15. Ghous M, Malik AN, Amjad MI, Kanwal M. High repetitions of Salat (prayer) activity in stroke rehabilitation. Rawal Med J. 2017; 42 (4): 604-605.

16. Ferreira LCM, Araujo AC, Oliveira CBS de, Jassi FJ, Oliveira VC, Negrão Filho R de F. Associação entre recrutamento de músculos abdominais com desfechos 
clínicos e risco prognóstico em indivíduos com dor lombar crônica não específica: estudo preliminar. Fisioter e Pesqui. 2016; 23 (1): 45-51. doi:10.1590/1809. 2950/14560723012016

17. Gong W. The effects of dynamic exercise utilizing PNF patterns on abdominal muscle thickness in healthy adults. J Phys Ther Sci. 2015; 27 (6): 1933-1936. doi: 10.1589/ipts.27.1933

18. Sharma V, Kaur J. Effect of core strengthening with pelvic proprioceptive neuromuscular facilitation on trunk, balance, gait, and function in chronic stroke. J Exerc Rehabil. 2017; 13 (2): 200-205. doi:10.12965/ jer. 1734892.446

19. Szczygieł E, Blaut J, Zielonka-Pycka K, et al. The Impact of Deep Muscle Training on the Quality of Posture and Breathing. J Mot Behav. 2018; 50 (2): 219-227. doi: 1
$0.1080 / 00222895.2017 .1327413$

20. Thabet AA, Alshehri MA. Efficacy of deep core stability exercise program in postpartum women with diastasis recti abdominis: a randomised controlled trial. J Musculoskelet Neuronal Interact. 2019; 19(1): 62-68.

21. Selkow NM, Eck MR, Rivas S. Transversus abdominis activation and timing improves following core stability training: a randomized trial. Int J Sports Phys Ther. 2017; 12 (7): 1048-1056. doi: 10.26603/ijspt20171048

22. Stepień A, Fabian K, Graff K, Podgurniak M, Wit A. An immediate effect of PNF specific mobilization on the angle of trunk rotation and the Trunk-Pelvis-Hip Angle range of motion in adolescent girls with double idiopathic scoliosis-a pilot study. Scoliosis Spinal Disord. 2017; 12: 29. doi:10.1186/s13013-017-0132-0 